\title{
Anatomía de la semilla de Casimiroa edulis (Rutaceae), "zapote blanco", durante su desarrollo
}

\author{
Hilda ARACELI ZAVALETA-MANCERA ${ }^{1,2}$ y E. MARK ENGLEMAN ${ }^{1}$
}

\begin{abstract}
RESUMEN. La semilla de la especie mexicana Casimiroa edulis Llave et Lexarza, es de interés por su uso medicinal. Se estudia su anatomía en las diferentes etapas de desarrollo desde óvulo hasta semilla madura. Se practicaron tinciones generales con safranina y verde fijo y particulares para detectar la presencia de almidón, lípidos, lignina, polifenoles y proteínas. Se evaluó el crecimiento del embrión y semilla con base en su volumen. El óvulo de $C$. edulis es singular; la falta de funículo, el hilo grande, la posición de la cálaza y la ligera curvatura de la nucela $\left(30-40^{\circ}\right)$ dificultan su clasificación. La semilla desarrolla una paquicálaza que ocupa alrededor del $70 \%$ de la superficie. La testa es delgada, no lignificada y rica en taninos. El embrión maduro ocupa alrededor del $90 \%$ del volumen de la semilla, desarrolla numerosas glándulas de aceite de origen lisígeno, es rico en granos de almidón y cuerpos de proteína. La radícula no apunta exactamente hacia el micrópilo; se ubica en el tercio dorsal micropilar. Casimiroa edulis se había descrito como carente de endospermo, pero en realidad la semilla madura tiene una pequeña cantidad de endospermo debajo de la paquicálaza.
\end{abstract}

ABSTRACT. The seed of the Mexican species, Casimiroa edulis Llave et Lexarza is valued for medicinal purposes. We have studied its anatomy in different stages of development from ovule to mature seed. We applied general staining with safranin and fast green and specific staining for the detection of starch, lipids, lignin, polyphenols, and proteins. Embryo and seed development was also evaluated on the basis of volume. The ovule is sessile, bitegmic, crassinucellate, hemianatropous, with a nucellar curvature of $30-40^{\circ}$ and a large hilum. The seed develops a large pachychalaza that covers approximately $70 \%$ of the surface. The testa is thin, nonlignified and tanniferous. The mature embryo occupies about $90 \%$ of the whole volume of the seed and contains lysigenous oil glands, starch grains and protein bodies. The radicle is

${ }^{1}$ Centro de Botánica, Colegio de Postgraduados, 56230 Montecillo, México.

2 Este trabajo representa parte de la tesis de Maestro en Ciencias, presentada en 1989 por la primera autora en el Colegio de Postgraduados, Montecillo, México.

Zavaleta-Mancera HA, Engleman E M. 1991. Anatomía de la semilla de Casimiroa edulis (Rutaceae), "zapote blanco", durante su desarrollo. Boletín de la Sociedad Botánica de México 51: 67-81. 
not directed exactly at the micropyle. It is located in the dorsal micropylar third of the seed. Casimiroa edulis had been described as lacking endosperm, but in reality the mature seed has a small amount of endosperm under the pachychalaza.

Casimiroa edulis Llave et Lexarza, comunmente llamado zapote blanco, es una de las diez especies que se citan para el género. Pertenece a la subfamilia Toddalioideae de la familia Rutaceae (Engler, 1931; Martínez, 1951; Chiang y González-Medrano, 1981 y Chiang, 1989). Es nativa de México; se encuentra silvestre, escapada o dentro del huerto familiar en zonas subtropicales y templadas hasta los $2500 \mathrm{~m}$ de altitud. Se distribuye principalmente desde el norte de México hasta Nicaragua, aunque también se ha introducido en Florida y California (USA), Brasil, la región del Mediterráneo y otros lugares (Popenoe, 1920; Martínez, 1951 y Ochse, et al., 1965).

El zapote blanco fue conocido por los aztecas bajo el nombre de cochitzápotl (cochi = dormir y tzápotl $=$ fruto carnoso dulce), aludiendo a sus propiedades somníferas (Códice Florentino, 1980, escrito aproximadamente entre 1560 y 1580). El protomédico Hernández (1959), apunta que los frutos son comidos para conciliar el sueño, que las hojas curan la diarrea de los niños y que la almendra es venenosa. Tras numerosos estudios realizados en el Instituto Médico Nacional, alrededor de 1898, el trabajo de Lille (1934) es el primero que corrobora experimentalmente el efecto hipotensor de los extractos de la semilla. Actualmente se propone la N,N-dimetilhistamina como la probable sustancia responsable de este efecto (Major y Dursch, 1958; Lozoya, et al., 1977, 1978; Lozoya y Enríquez, 1981). La medicina tradicional mexicana actualmente recurre al uso de hojas y semillas del zapote blanco para bajar la presión arterial (Lozoya y Enríquez, 1981).

Poco se conoce acerca de la anatomía y morfología de la semilla. Schroeder (1954) escribe sólo sobre la anatomía del fruto. Por otra parte, la estructura fibrosa que cubre la semilla es referida como endocarpo por Engler (1931) y Schroeder (1954), y como testa fibrosa por Bickerm en 1897 (citado por Netolitzky, 1926) y Corner (1976). Para los primeros el fruto es una drupa, y para los segundos es una baya.

Este estudio se propuso tomando en cuenta la importancia alimenticia y medicinal que tiene Casimiroa edulis, ya que la información sobre la estructura de la semilla es escasa y confusa.

\section{MATERIALES Y MÉTODOS}

Se colectó en La Garita, Mpio. de Jungapeo, Michoacán (19² $28^{\prime} 50^{\prime \prime}$ N y $100^{\circ} 29^{\prime}$ 14 " W), lugar subtropical en donde abunda el zapote blanco como cerco vivo y dentro del huerto familiar. Del 20 de diciembre de 1987 al 8 de mayo de 1988 se hicieron ocho colectas periódicas en un árbol adulto (20 años), de aproximadamente $10 \mathrm{~m}$ de alto, 
obteniéndose desde flores hasta frutos maduros. Los frutos se agruparon en clases o intervalos con base en sus diámetros ecuatoriales, con una relación de 0.75 entre un intervalo y el subsecuente. Con el promedio de los logarítmos de los diámetros de los frutos colectados en cada fecha, se elaboró la curva de crecimiento. Para mayor detalle véase Zavaleta-Mancera y Engleman (1991). Con la ayuda de la gráfica se estimaron las edades de cada clase:

$\begin{array}{lll}\text { Clase } 1 \text { ( 0-8 días) } & \text { Clase } 5 \text { (32-40 días) } & \text { Clase } 9 \text { ( 71-90 días) } \\ \text { Clase } 2 \text { ( } 8-16 \text { días) } & \text { Clase } 6 \text { (40-48 días) } & \text { Clase } 10 \text { ( } 90-113 \text { días) } \\ \text { Clase } 3 \text { (16-24 días) } & \text { Clase } 7 \text { (48-57 días) } & \text { Clase } 11 \text { (113-175 días) } \\ \text { Clase } 4 \text { (24-32 días) } & \text { Clase } 8 \text { (57-71 días) } & \end{array}$

Para el estudio estructural, parte del material se fijó en Craf III o en FAA (Sass, 1958) para su inclusión y corte en parafina. Los cortes así obtenidos se tiñeron con safranina 0-verde fijo FCF. En el estudio histoquímico, se realizaron las siguientes tinciones específicas: de lípidos con rojo 0 de aceite $(0.05 \% \mathrm{p} / \mathrm{v}$ rojo 0 de aceite $+25 \%$ $\mathrm{v} / \mathrm{v}$ de 1-butanol $+75 \%$ v/v etilenglicon (E. Mark Engleman, comunicación personal); en almidón con yodo más yoduro de potasio (Johansen, 1940); de proteína con azul negro de naftol (Fisher, 1968); de lignina con floroglucinol y ácido clorhídrico (Sass, 1958); de polifenoles (leucoantocianinas y catequinas) con vainillina-ácido clorhídrico (Gardner, 1975) y cloruro férrico Reeve en 1959b (citado por Jensen, 1962). Además se disoció el endocarpo con solución Jeffrey (Johansen, 1940).

Se elaboró una curva de crecimiento para la semilla y otra para el embrión graficando el logaritmo base 10 del volumen contra el tiempo. El cálculo del volumen supone que la forma de la semilla y embrión es aproximadamente elipsoidal durante todo el desarrollo. Se usó la fórmula del elipsoide $V=4 / 3 \pi$ a b c , donde a $=1 / 2$ largo, $b=$ $1 / 2$ ancho y $c=1 / 2$ grueso; donde $a, b, c$, son las longitudes de los semiejes.

Se calcularon los cambios de redondez de la semilla, mediante el cociente de ancho/grueso. El grueso se midió en el corte trasversal de la semilla en el sentido radial con respecto al fruto y el ancho en el sentido tangencial.

\section{RESULTADOS}

El ovario posee cinco óvulos con placentación axial. Rodeando cada lóculo se observó una red de tejido vascular que en la madurez forma parte del endocarpo (fig. 1).

1. Ovulo en antesis. El óvulo es ovoide, sésil, bitégmico, crasinucelado y con un hilo grande. La nucela tiene forma de un cono con la base oblicua. La nucela es ligeramente 
curva porque el eje de la punta (A-A') y el eje de la base (B-B') forman aproximadamente un ángulo de $30-40^{\circ}$ (figs. 2,5 ).

El micrópilo está formado sólo por el tegumento interno. Junto al micrópilo, se observó una protuberancia masiva (fig. 3) que, en algunos cortes, parece tener mayor contacto con la placenta del fruto y en otros cortes, con el tegumento externo. En varios cortes se observó una clara continuidad de las hileras celulares entre el estigma y esta estructura. Algunas células de la protuberancia exhiben citoplasma denso y núcleos grandes, típico de células glandulares. Esta estructura persiste después de la fecundación y aparece como un pequeño resto papiráceo en la semilla madura.

Se estudiaron cortes seriados de cuatro sacos embrionarios de ovarios de 3.2-4.2 mm de diámetro. En tres sacos se observaron uno o dos núcleos de la célula central y dos sinérgidas. En el cuarto saco se observaron un núcleo central, dos sinérgidas, la ovocélula y dos antípodas (fig. 4).

El tegumento interno consta de tres a cuatro estratos de células isodiamétricas, de pared delgada y sin taninos. El tegumento externo en forma de cono, es rico en taninos condensados; tiene en promedio 11 estratos de grueso (fig. 2).

El haz vascular que irriga el óvulo y la semilla entra aproximadamente por la parte media del hilo (figs. 2, 5). Este penetra en el óvulo hasta llegar a la cálaza, extensa y en forma de plato, la cual ocupa aproximadamente $40 \%$ del contorno de la nucela en el plano mediano (figs. 2, 5). Entre la nucela y la región vascular de la calaza existe una capa muy evidente de células con abundantes taninos condensados.

2. Semilla. Morfología de la semilla madura. Al quitar el endocarpo fibroso, se revela la semilla madura, que es ovoide y ligeramente aplanada en una de sus caras. Mide de 3 a $4 \mathrm{~cm}$ de largo y de 1.5 a $2.5 \mathrm{~cm}$ de ancho y grueso. En ella se observan, en perfil, dos contornos opuestos; uno es el hilo de forma recta y otro es el contorno convexo de la semilla que por razones de exposición los hemos denominado como región ventral y dorsal respectivamente (fig. 6).

La semilla es sésil con un hilo largo que se extiende de un extremo a otro. La cubierta seminal es lisa, delgada, lustrosa y de color pardo claro; en ella se distinguen dos zonas:

1) Una zona de color claro, que corresponde a la paquicálaza (fig. 6A), de aproximadamente $1.0 \mathrm{~mm}$ de grueso, que se extiende de la región ventral (cerca del hilo) hacia la dorsal (lejos del hilo), ocupando aproximadamente el $70 \%$ de la superficie de la semilla.

2) Otra región de color más oscuro que la anterior, que corresponde a la testa libre (fig. 6A) de aproximadamente $0.2 \mathrm{~mm}$ de grueso, que ocupa el $30 \%$ restante.

El embrión es carnoso y de color blanco y ocupa el $86 \%$ del volumen de la semilla. Está formado por dos cotiledones grandes y gruesos, una radícula corta y una plúmula con algunos primordios de hoja. La radícula no siempre apunta hacia el micrópilo; comúnmente se encuentra en el primer tercio micropilar del dorso de la semilla (fig. 6B). 


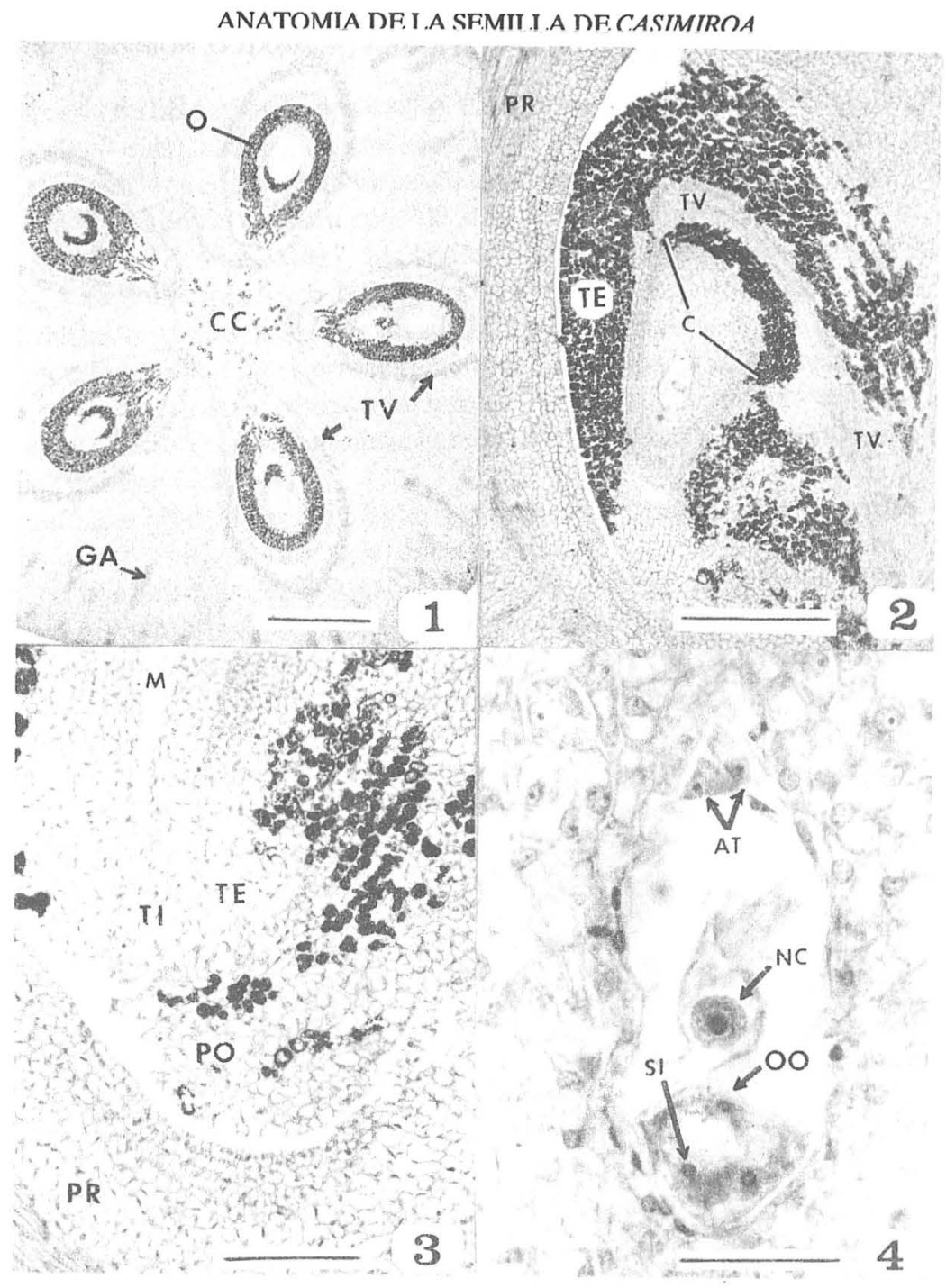

Fig. 1. Sección transversal de un ovario en antesis (clase 1), con cinco lóculos y la placentación axial. $0=$ Ovulo; TV $=$ Red de tejido vascular; $\mathrm{CC}=$ Cuerpo Central; $\mathrm{GA}=$ Glándula. Barra $=500 \mu$.

Fig. 2. Corte mediano del óvulo en antesis (clase 1). TE = Tegumento externo; $\mathrm{C}=$ Cálaza; $\mathrm{TV}=\mathrm{Haz}$ vascular; $\mathrm{PR}=$ Pericarpo. Barra $=300 \mu$.

Fig. 3. Corte mediano de la protuberancia micropilar (PO) (clase 1). $M=$ Mirópilo; PR = Pericarpo; TE $=$ Tegumento externo; $\mathrm{TI}=$ Tegumento interno. Barra $=100 \mu$.

Fig. 4. Corte longitudinal del saco embrionario (clase 1). SI = Núcleo de sinérgida; $00=$ Núcleo de la ovocélula; $\mathrm{NC}=$ Núcleo de la célula central $; \mathrm{AT}=$ Antípodas. Barra $=30 \mu$. 
A

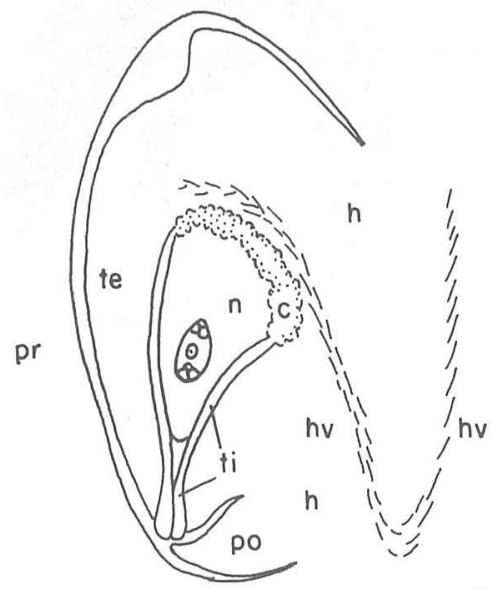

Fig. 5
B

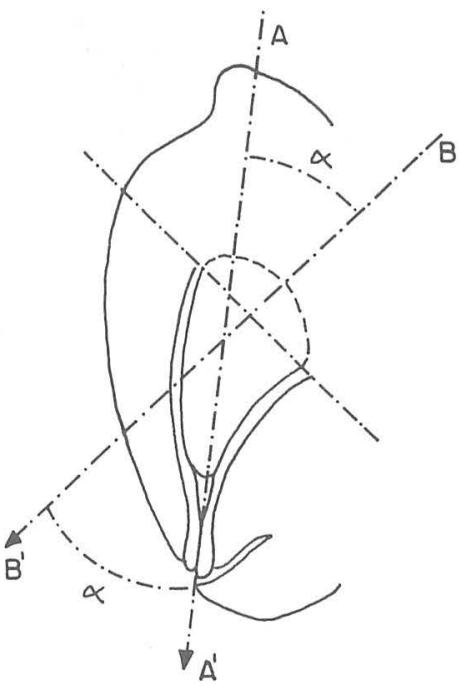

A

B

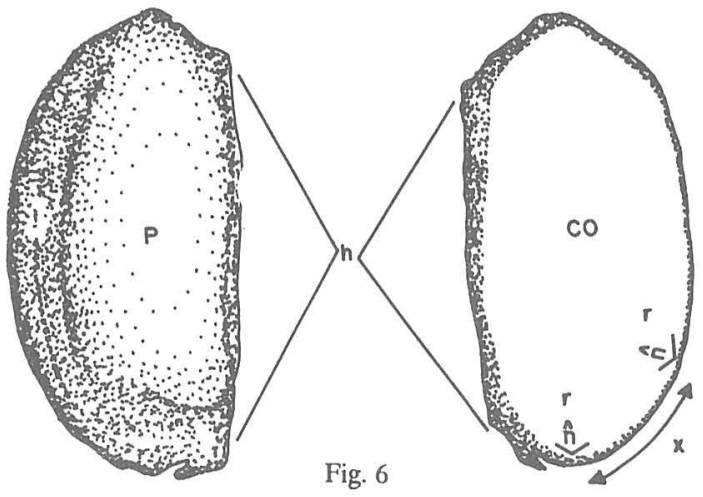

Fig. 5. Ovulo en antesis, comparable a la figura 2A) Corte mediano del óvulo. $\mathrm{n}=$ nucela; te $=$ tegumento externo; $\mathrm{ti}=$ tegumento interno, $\mathrm{po}=$ protuberancia; $\mathrm{c}=$ cálaza; $\mathrm{h}=$ hilo; $\mathrm{hv}=$ haz vascular; $\mathrm{pr}=$ pericarpo (fruto). B) Curvatura de la nucela. $\mathrm{A}-\mathrm{A}^{\prime}=$ eje de la punta de la nucela; $\mathrm{B}-\mathrm{B}^{\prime}=$ eje de la base de la nucela; $\alpha=$ curvatura de la nucela.

Fig. 6. Semilla madura. A) Vista externa. $\mathrm{h}=$ hilo; $\mathrm{p}=$ paquicálaza; $\mathrm{t}=$ testa (tegumento). $\mathrm{B}$ ) Corte Mediano. $c o=$ cotiledón $; \mathrm{r}=$ radícula; $\mathrm{x}=$ posición probable de la radícula. 
Anatomía de la semilla en desarrollo. Cuando la semilla alcanza en promedio $6 \mathrm{~mm}$ de largo (40-48 días), el embrión aún es globular y mide de 70 a $100 \mu$ de diámetro (figs. $7,8)$. El suspensor es corto y está formado de algunas células isodiamétricas. Posteriormente pasa al estado de corazón con 200 u de diámetro en promedio.

Luego (clase 7, 48-57 días), los cotiledones crecen rápidamente y el suspensor degenera. Los cotiledones son hojas carnosas y el eje embrionario es corto. Pueden distinguirse los meristemos apicales (radicular y de vástago) y el procámbium (fig. 9).

Cuando la semilla mide aproximadamente $11 \mathrm{~mm}$ de largo (clase 8, 57-71 días), la radícula no se ubica en su posición micropilar debido al crecimiento diferencial de los cotiledones. Así en la semilla madura, la radícula se localiza a lo largo del primer tercio micropilar del dorso (fig. 6).

Cuando la semilla alcanza 6.2 \% de su volumen final (clase 9,71-90 días), el embrión ocupa toda la semilla. Se inicia la diferenciación de glándulas de aceite, de origen lisígeno, en la epidermis cotiledonar (fig. 10). Después aparecen numerosas glándulas de aceite distribuidas en todo el embrión (fig. 11), incluyendo la plúmula. Simultáneamente, aparecen granos de almidón cerca de los núcleos de las células del parénquima fundamental. En la madurez los granos de almidón llenan casi por completo el lumen celular.

Cuando la semilla tiene $30 \%$ de su volumen final (clase 10, 90-113 días), el embrión posee una plúmula con algunos primordios de hojas, con glándulas de aceite. Simultáneamente se inicia la acumulación de cuerpos de proteína en el parénquima cotiledonar. En la semilla madura la proteína es menos abundante que el almidón (fig. 12).

En el embrión maduro los lípidos se encuentran principalmente en las glándulas de aceite, cerca del tejido vascular y en la epidermis, mientras que el resto del tejido contiene escasos esferosomas.

En la semilla madura, los polifenoles (taninos) se ubican principalmente en la cubierta seminal. Los cotiledones presentaron una respuesta muy leve y a veces negativa a la tinción para polifenoles.

Por la ausencia casi completa del endospermo, el embrión, principalmente los cotiledones, constituye la fuente principal de reservas de la semilla madura. Este embrión contiene numerosos granos de almidón, cuerpos de proteína (fig. 12), y numerosas glándulas de aceite de origen lisígeno especialmente en el lado adaxial de los cotildones (fig. 11).

El embrión de Casimiroa edulis presenta su máxima tasa relativa de crecimiento aproximadamente entre los 32 y 65 días después de la antésis (fig. 15). En este período, el embrión pasa del estado globular al cotiledonar.

Durante el desarrollo la semilla no sólo aumenta de tamaño sino que además cambia de forma. La redondez, estimada como ancho/grueso, se mantiene más o menos constante con un valor de 0.58 durante los primeros 86 días. A partir de esta edad el valor sube a 0.89 en la madurez. 
Endospermo y nucela. El endospermo exhibe un tipo de desarrollo nuclear. Las paredes se forman cuando el embrión pasa del estado globular al de corazón (fig. 8). Entre los 57 y 71 días el endospermo es completamente celular y rico en lípidos.

Con el crecimiento del embrión, el endospermo se reduce rápidamente. En la semilla madura éste se encuentra como pequeños manchones de uno a dos estratos de células intactas, ricas en lípidos, debajo de la paquicálaza.

Cubierta seminal. La cubierta seminal madura es delgada y no lignificada. Está compuesta por la testa y la paquicálaza.

En antesis el tegumento interno (TI) tiene en promedio cuatro estratos de parénquima sin taninos condensados. El tegumento externo (TE), tiene en promedio de 8-9 estratos de parénquima con abundantes taninos condensados que corresponden a la exotesta (1 estrato) y mesotesta (8-9 estratos). La endotesta tiene 1-2 estratos sin taninos condensados (fig. 13). Sin embargo la vanillina- $\mathrm{HCl}$ evidenció la presencia de taninos solubles en toda la endotesta y (TI).

Durante el desarrollo la exotesta, que consta de un estrato, siempre divide anticlinalmente. La mesotesta presenta pocas divisiones periclinales. La endotesta se divide mucho en todos los sentidos, de tal manera que a los 57-71 días (clase 8) el TE alcanza su máximo grueso con 41 estratos en promedio; de los cuales 11-13 estratos externos corresponden a la exotesta y mesotesta y los 28-30 restantes son endotesta, sin taninos condensados. A esta edad el TI se ha aplastado por completo (figs. 14, 16). Después la endotesta se aplasta totalmente.

Finalmente la testa madura (fig. 17) queda formada con 12 estratos en promedio de células muertas, de paredes delgadas, no lignificadas, con taninos condensados, aplastadas en plano tangencial y separadas por espacios intercelulares. La apariencia en corte es la de un encaje.

Durante el desarrollo la estructura de la paquicálaza básicamente no se modifica, sólo se extiende y engruesa. En un corte mediano la cálaza en antesis ocupa aproximadamente el $40 \%$ del contorno nucelar (figs. 2,5); después la cálaza se extiende hasta ocupar aproximadamente el $70 \%$ de la superficie de la semilla madura (fig. 6A).

Debajo de la paquicálaza madura se observaron pequeños manchones de uno o dos estratos de células intactas ricas en lípidos, que corresponden a restos de endospermo (EN) (figs. 18, 19).

En la paquicálaza madura (fig. 18) pueden distinguirse de afuera hacia adentro: a) La epidermis externa, o exotesta $(E)$, formada de un solo estrato de células ricas en taninos condensados. b) Una capa externa de 15 a 20 estratos de células con paredes delgadas, no lignificadas, con taninos condensados (TA) y separadas por espacios intercelulares. c) Una red de tejido vascular (TV) con xilema y floema. d) Una capa formada de 5 a 7 estratos de células con abundantes taninos condensados en masas coherentes (TA). e) Una región interna (N) constituida por células grandes, con paredes delgadas, las cuales comparten características con las de la nucela. Aquí se observaron lípidos en las aristas de sus células. Estos depósitos se observaron en material fresco. 
ANATOMIA DE LA SEMILLA DE CASIMIROA

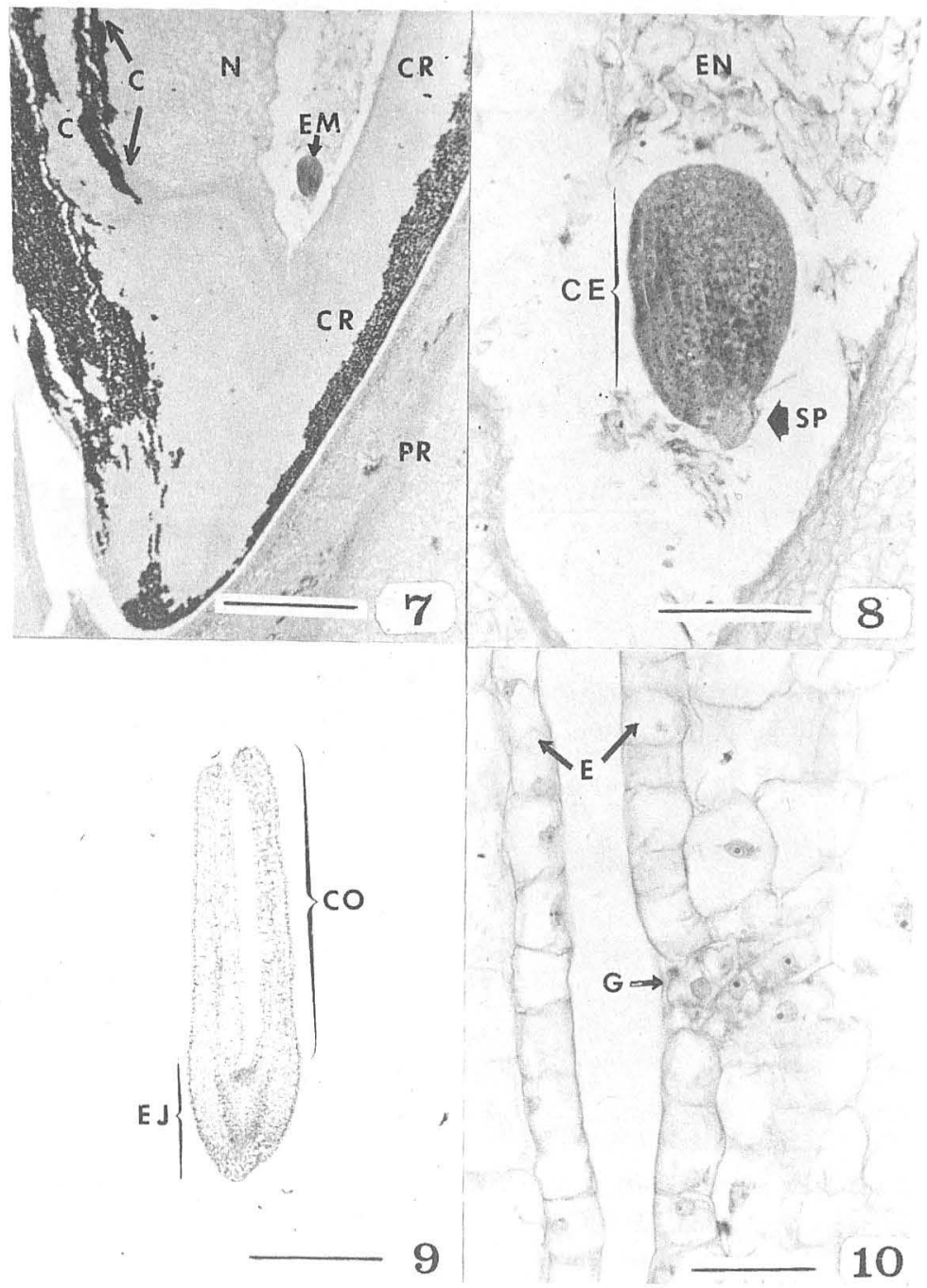

Fig. 7. Corte longitudinal de la semilla (clase 6). $\mathrm{EM}=$ Embrión en estado globular; $\mathrm{N}=$ Nucela; $\mathrm{C}=$ Cálaza; $\mathrm{CR}=$ Cubierta seminal; $\mathrm{PR}=\mathrm{Barra}=500 \mu$.

Fig. 8. Aumento del embrión de la figura 7. $\mathrm{SP}=$ Suspensor; $\mathrm{CE}=$ Cuerpo del embrión; $\mathrm{EN}=$ Endospermo. Barra $=100 \mu$.

Fig. 9. Corte longitudinal de un embrión cotiledonar (clase 7). Nótese el procámbium en el eje embrionario (EJ) y en un cotiledón (CO). Barra $=400 \mu$.

Fig. 10. Primordio de una glándula de aceite en la epidermis superior cotiledonar (clase 9), corte transversal. $\mathrm{G}=$ Primordio de glándula; $\mathrm{E}=$ Epidermis cotiledonar. Barra $=40 \mu$. 


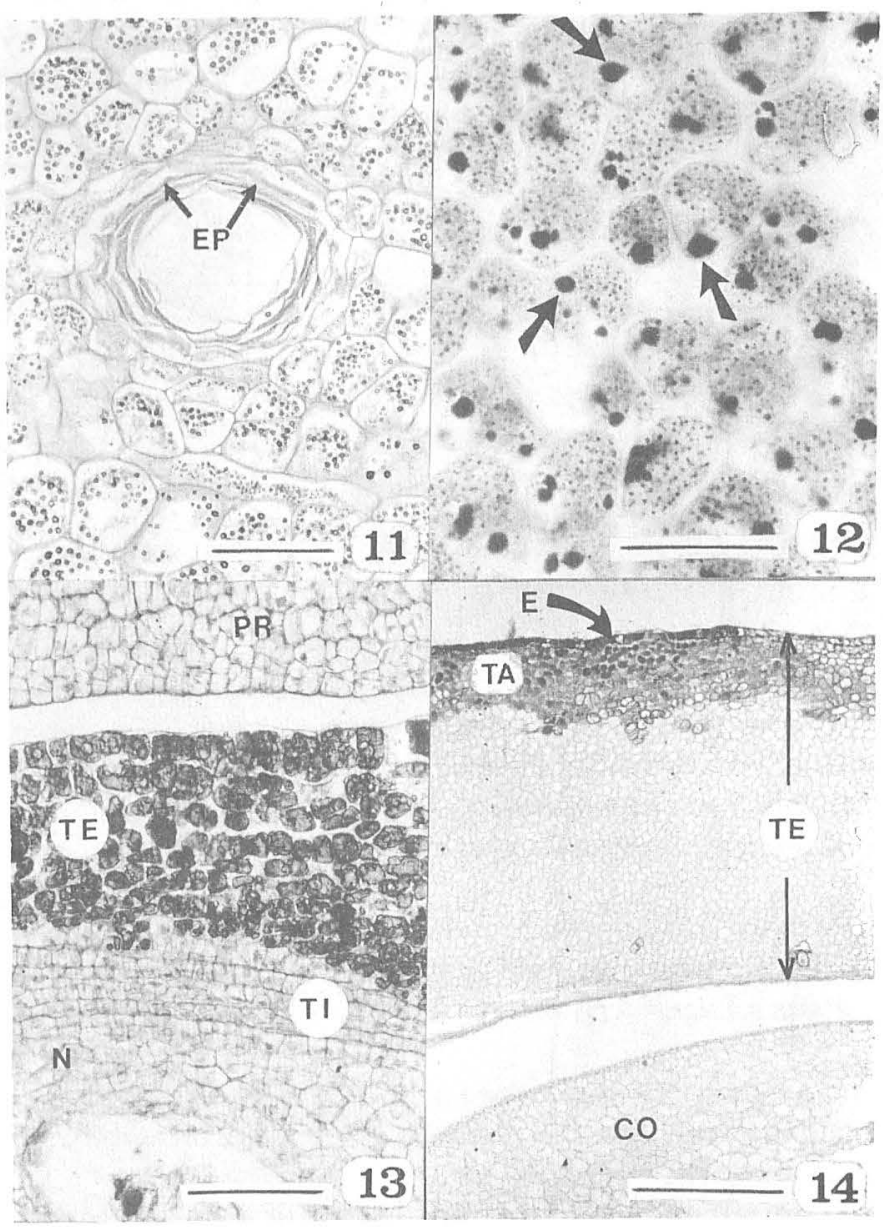

Fig. 11. Glándula de aceite madura en corte transversal de cotiledón. Los puntos negros son espacios de aire en los granos de almidón. EP $=$ Células epiteliales. Barra $=100 \mu$.

Fig. 12. Cuerpos de proteína de las células del cotiledón maduro. Corte transversal teñido con azul negro de naftol. Las flechas indican los cuerpos de proteína y los puntos pequeños oscuros son espacios de aire en los granos de almidón. Barra $=100 \mu$.

Fig. 13. Ovulo en antesis (clase 1). Corte mediano con el micrópilo hacia el lado izquierdo. Nótese los abundantes taninos condensados en el tegumento externo (TE). TI = Tegumento interno; $\mathrm{N}=$ Nucela; $\mathrm{PR}=$ Pericarpo. Barra $=70 \mu$.

Fig. 14. Cubierta seminal (clase 8). Corte longitudinal con el micrópilo hacia el lado derecho. Nótese el aumento en número de células del tegumento externo (TE), con respecto a la figura 15 . $\mathrm{E}=\mathrm{Epidermis}$ externa (exotesta); $\mathrm{TA}=$ Capa con taninos (región externa de la mesotesta); $\mathrm{CO}=$ Cotiledón. Barra $=$ $300 \mu$. 

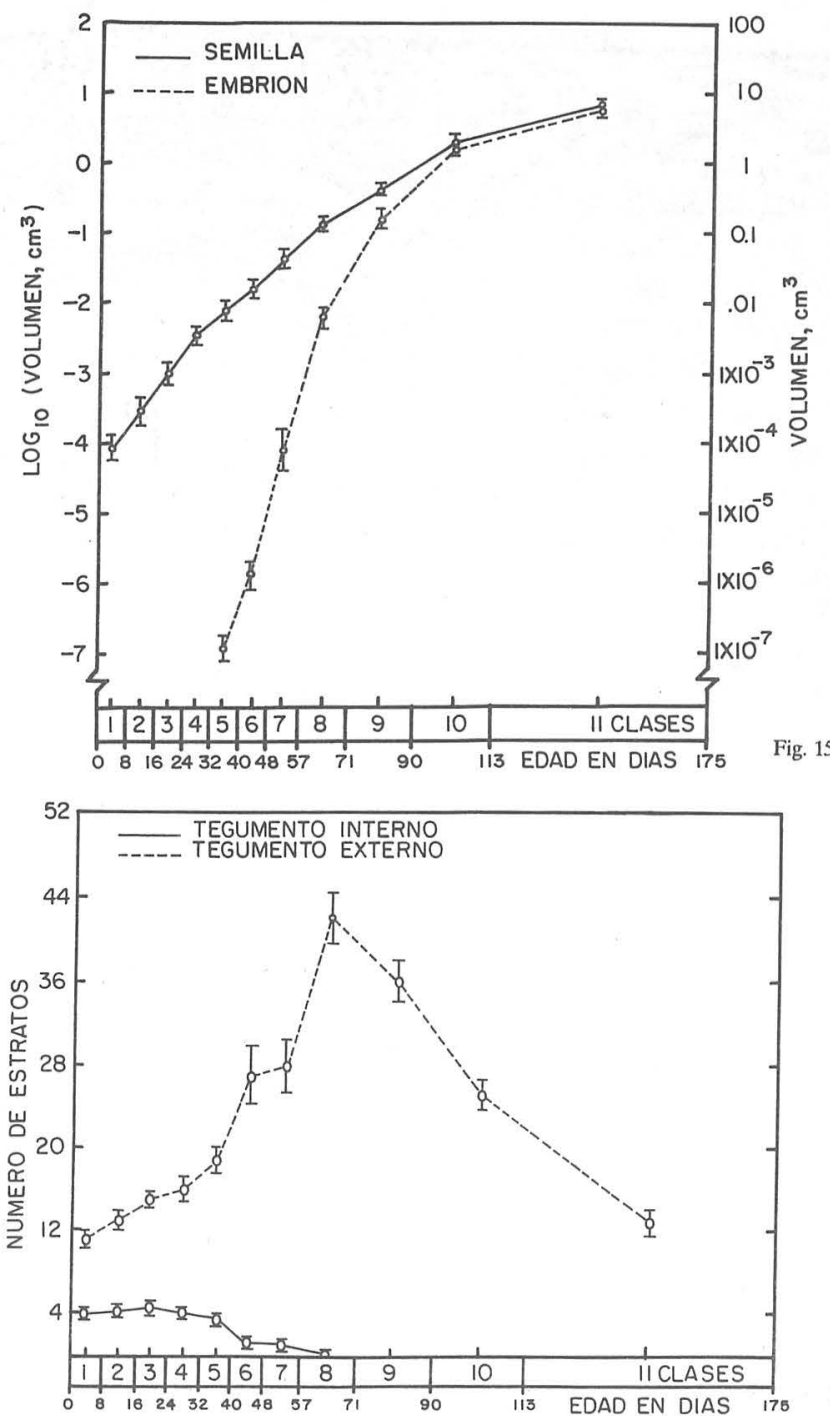

Fig. 16

Fig. 15. Curva de crecimiento del embrión y la semilla. Los valores son promedios de log (10) del volumen el error estándar de la media. Las clases se basan en el diámetro del fruto.

Fig. 16. Grueso en número de estratos del tegumento externo e interno, durante el desarrollo. Los valores son el promedio de ocho mediciones por clase el error estándar de la media. Las clases se basan en el diámetro del fruto. 

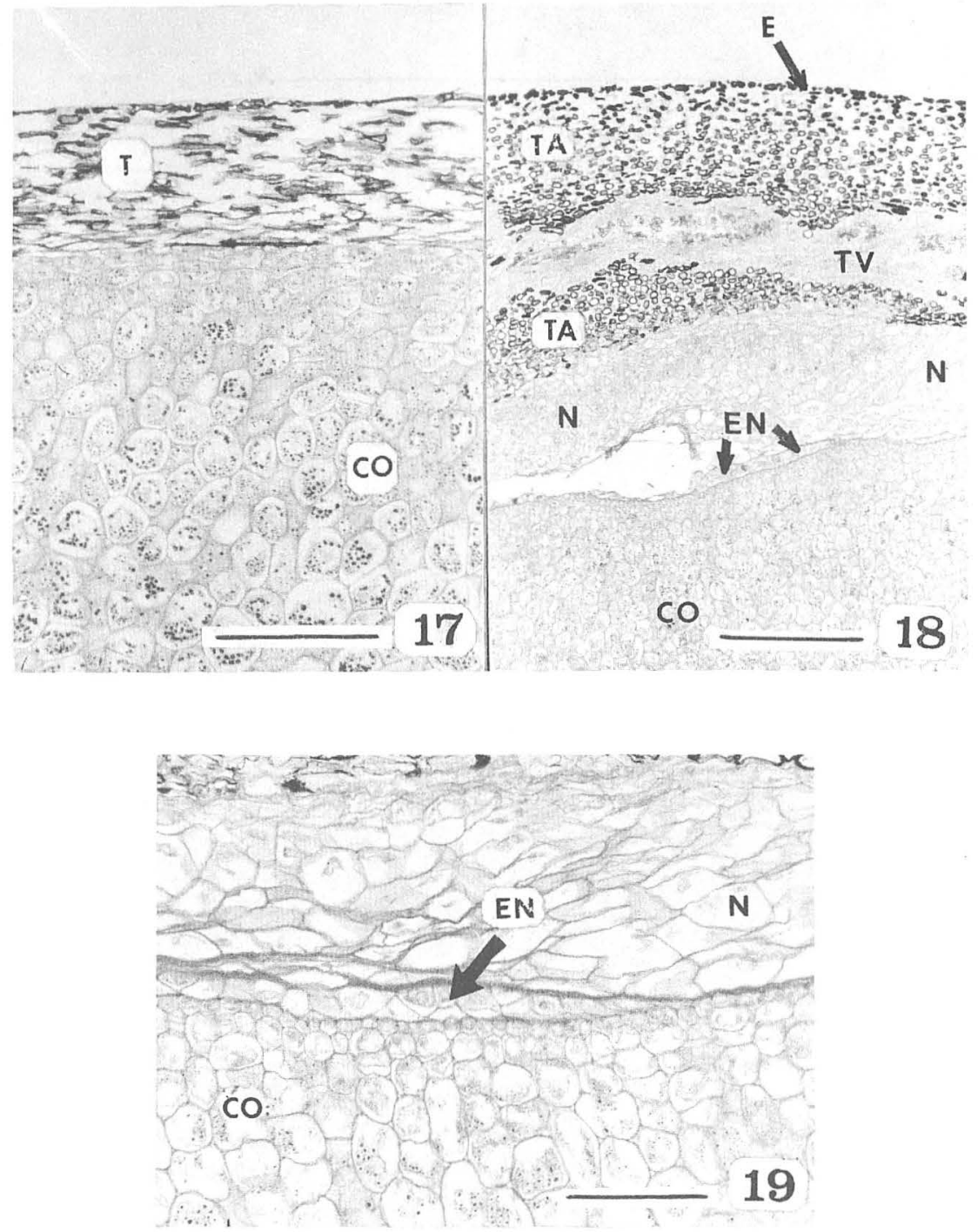

Fig. 17. Testa madura (corte transversal). Nótese la apariencia de encaje de la testa (T). CO = Cotiledón. Barra $=200 \mu$.

Fig. 18. Paquicálaza de la semilla madura en corte transversal. Nótese la posición de los restos de endospermo $(\mathrm{EN})$. $\mathrm{E}=$ Epidermis externa (exotesta); $\mathrm{TA}=$ Tejido rico en taninos; $\mathrm{TV}=$ Red de tejido vascular; $\mathrm{N}=$ Región interna sin taninos; $\mathrm{CO}=$ Cotiledón. Barra $=400 \mu$.

Fig. 19. Aumento de los restos de endospermo de la figura 18. $\mathrm{N}=$ Región interna $\sin$ taninos; $\mathrm{CO}=$ Cotiledón; $\mathrm{EN}=$ Restos de endospermo. Barra $=100 \mu$. 


\section{DISCUSIÓN Y CONCLUSIONES}

El óvulo de Casimiroa edulis, como el de la mayoría de las rutáceas, es bitégmico, crasinucelado, con endospermo de tipo nuclear.

El criterio tomado en el trabajo para describir la curvatura del óvulo es el de tomar como base la curvatura de la nucela. Esta curvatura se midió tomando como referencia el eje de la base y el eje de la punta de la nucela. Con este criterio la nucela de Casimiroa edulis tiene una curvatura de $30-40^{\circ}$ sin que el óvulo llegue a ser campilótropo.

La información sobre la curvatura de la nucela en los óvulos de las rutáceas no es un tema muy concurrido. Por una parte Boesewinkel (1977) menciona que en Skimmia japonica la curvatura del óvulo excede los $90^{\circ}$, pero el óvulo no llega a ser campilótropo. Tomando como referencia el eje de la base y el eje de la punta de la nucela medimos la curvatura de la nucela de los óvulos descritos por Boesewinkel (1977). Encontramos que la nucela de Ruta graveolens tiene $30^{\circ}$ de curvatura, Skimmia japonica $40^{\circ}$ y Zanthoxylum simulans tiene la nucela recta. Esto sugiere que sería interesante revisar otros géneros de la familia.

Si se considera que el centro del hilo es el lugar en donde entra el haz vascular, es posible reconocer una rafe, entre el punto de entrada del haz vascular y el centro de la paquicálaza. Con estas consideraciones el óvulo tiende a ser hemianátropo con una rafe corta.

Con base en las observaciones realizadas en este trabajo y lo citado por Davis (1966), es probable que el saco embrionario de $C$. edulis sea el tipo Polygonum, a reserva de un estudio más detallado sobre su ontogenia.

Algunas características de la protuberancia observada en el óvulo de C. edulis, como su posición micropilar, características glandulares de algunas de sus células, continuidad de ciertas hileras celulares entre la protuberancia y el tejido estigmático, consistencia papirácea y tamaño pequeño en semilla madura, y semejanza con el obturador de Zanthoxylum simulans descrito (Boesewinkel, 1977), sugieren que esta protuberancia micropilar es un obturador. Un estudio minucioso de la ontogenia del óvulo y de la fase progámica permitiría conocer con certeza la naturaleza de esta estructura.

Power y Callan (1911); Kincl, et al. (1956) y Enriquez, et al. (1984) informaron de la presencia de sustancias fenolicas en la semilla de Casimiroa, pero no detallaron su ubicación. Nuestras pruebas para polifenoles los localizaron principalmente en la cubierta seminal.

En oposición a los trabajos de Bickerm de 1897 (citado por Netolitzky, 1926) y de Corner (1976), los resultados de este estudio indican que la testa es delgada, papirácea, no lignificada y rica en taninos. La estructura fibrosa que cubre la semilla es un endocarpo en lo que concordamos con Engler (1931) y Schroeder (1954), y no una testa como Corner (1976) y otros autores piensan. 
Entre las semillas de la subfamilia Toddalioideae descritas por Corner (1976) y Boesewinkel (1977), C. edulis es muy parecida/a Skimmia en cuanto al desarrollo y estructura de la testa. Sin embargo el óvulo de Skimmia es anátropo mientras que el de Casimiroa se aproxima a hemianátropo. Casimiroa, a diferencia de Skimmia, desarrolla una paquicálaza que llega a ocupar alrededor del $70 \%$ de la superficie de la semilla. Consideramos que la presencia de una extensa cálaza en C. edulis obedece a la necesidad de suministrar suficientes nutrientes a la semilla durante su rápido crecimiento.

Según las descripciones de Glycosmis arborea (Aurantioideae), hechas por Boesewinkel y Bouman (1978), y las observaciones hechas en Casimiroa edulis, ambos géneros muestran paquicálazas, estructuralmente muy semejantes, con la diferencia de que en la región más interna de la paquicálaza de $C$. edulis existen lípidos solubles en las aristas celulares.

Según Engler (1931) Casimiroa y Sargentia recientemente transferida a Casimiroa por Chiang (1989), carecen de tejido de reserva, aunque los demás géneros de la subtribu Toddaliinae lo tienen. Nuestro hallazgo de pequeñas cantidades de endospermo, sugiere que la diferencia es meramente cuantitativa.

Las semillas de la subfamilia Toddalioideae exhiben una amplia gama de variación en la estructura de sus testas, que va desde la testa compleja y lignificada de Toddalia hasta las testas sencillas e indiferenciadas de Skimmia y Casimiroa. Esta gama de variación confiere valor taxonómico a la anatomía de la cubierta seminal en el estudio de esta subfamilia.

AGRADECIMIENTOS. Agradecemos al Consejo Nacional de Ciencia y Tecnología (CONACYT), por su apoyo a la primera autora mediante una beca de maestría. A Benjamín Zavaleta Rodríguez y Humberto López Delgado por su colaboración en el trabajo de campo. Al Dr. Raúl Enríquez Habib del Instituto đe Química, UNAM y a la Dra. Judith Márquez Guzmán de la Facultad de Ciencias, UNAM por sus valiosos comentarios y a dos revisores anónimos por los comentarios hechos al trabajo.

\section{LITERATURA CITADA}

BOESEWINKEL, F.D. 1977. Development of ovule and testa in Rutaceae. I. Ruta, Zanthoxylum, and Skimmia. Acta Bot. Neerl. 26(3):193-211.

BoESEWINKEL, F.D. y F. BOUMAN. 1978. Development of ovule and testa in Rutaceae. II. The unitegmic and pachychalazal seed of Glycosmis arborea (Roxb.) D.C. Acta Bot. Neerl. 27(1):68-78.

ChiAnG, F. y F. GonZÁLEZ-MEDRANO. 1981. Nueva especie de Casimiroa (Rutaceae) de la zona árida oaxaqueño-poblana. Bol. Soc. Bot. México 41:23-26.

ChIANG, F. 1989. Casimiroa greggii, formerly in Sargentia (Rutaceae). Taxon 38:116-119.

CODICE FLORENTINO. 1980. Edición facsímil del manuscrito 218-20 de la Colección Palatina de la Biblioteca Medicea Laurenziana. Gobierno de la República Mexicana. 3v.

CORNER, E.J.H. 1976. The seeds of dicotyledons. Cambridge University Press, London. 2v.

DAVIS, G.L. 1966. Systematic embryology of the angiosperms. Wiley, New York. 528p. 
ENGLER, A. 1931. Rutaceae. En: Engler, A. y H. Harms. Die natürlichen Pflanzenfamilien. 2a. ed. Band 19a:187-359.

ENRIQUEZ, R.G., M.L. ROMERO, L.I. ESCOBAR, P. JOSEPH-NATHANy W.F. REYNOLDS. 1984. High-performance liquid chromatographic study of Casimiroa edulis. II. Determination of furocoumarins. J. Chromatography 287:209-214.

FISHER, D.B. 1968. Protein staining of ribboned Epon sections for light microscopy. Histochemie 16:92-96.

GARDNER, R.O. 1975. Vainillin hydrochloric acid as histochemical test for tannin. Stain Technol. 50:315-318.

HERNÁNDEZ, F. 1959. Historia de las plantas de la Nueva España. (Traducido del latín de la versión de 1790). Universidad Nacional Autónoma de México, México. 3v.

JENSEN, W.A. 1962. Botanical histochemistry. Principles and practice. Freeman, San Francisco. 408p.

JOHANSEN, D.A. 1940. Plant microtechnique. McGraw-Hill, New York. 503p.

KINCL, F.A., J. ROMO, G. ROSENKRANZy F. SONDHEIMER. 1956. The constituents of Casimiroa edulis Llave et Lex. Part I. The seed. J. Chem. Soc. 4:4163-4169.

LILLE, J. DE. 1934. Nota acerca de la acción del zapote blanco sobre la presión arterial. An. Inst. Biol. Univ. Nac. Autón. México 5(1):45-47.

LozoYA, X., G. RoMERO, M. OlmEdo y A. BONDANI. 1977. Farmacodinámica de los extractos alcohólico y acuoso de la semilla de Casimiroa edulis Arch. Invest. Méd. (México) 8:145-150.

LozoyA, X., D. RodrígueZ-REYnAGA, J. ORTEGA-GALVÁN y R. EnRÍQueZ-HABIB. 1978. Aislamiento de una substancia hipotensora de la semilla de Casimiroa edulis. Arch. Invest. Méd. (México) 9:565-573.

LOZOYA, X., y R. ENRÍQUEZ. 1981. El zapote blanco. Consejo Nacional de Ciencia y Tecnología, México. $134 \mathrm{p}$.

MAJOR, R.T., y F. DURSCH. 1958. N,N-dimethylhistamine, a hypotensive principle in Casimiroa edulis Llave et Lex. J. Org. Chem. 23: 1564-1565.

MARTíneZ, M. 1951. Las casimiroas de México y Centroamérica. An. Inst. Biol. Univ. Nac. Autón. México 22(1):25-81.

NetOLITZKY, F. 1926. Anatomie der Angiospermen-Samen. Handbuch der Pflanzenanatomie. Borntraeger, Berlin, 364p.

OCHSE, J.J., M.J. JOULE, y J.M. DIJKMAN. 1965. Cultivo y mejoramiento de plantas tropicales y subtropicales. LIMUSA, México. 2v.

POPENOE, W. 1920. Manual of tropical and subtropical fruits. Hafner Press, Collier, New York. 474p.

POWER, F.B., y T. CALLAN. 1911. The constituents of the seed of Casimiroa edulis. J. Chem. Soc. 99:1993-2011.

SASS, J.E. 1958. Botanical microtechnique. 3a. ed. Iowa State University Press, Ames, Iowa. 277p.

SCHROEDER, C.A. 1954. Fruit morphology and anatomy in white sapote. Bot. Gaz. 115:248-254.

ZavaletA-MANCERA, H.A. y E. M. ENGLEMAN. 1991. Anatomía del fruto de Casimiroa edulis (Rutaceae), "zapote blanco", durante su desarrollo. Bol. Soc. Bot. México 51:53-65. 